Original Research Paper

\title{
Rotation Center Fitting based on Rotation Hybrid Calibration Method
}

\author{
${ }^{1}$ Yi Guo, ${ }^{2}$ Doudou Lei, ${ }^{2}$ Yu Liu and ${ }^{2}$ Baolong Liu \\ ${ }^{1}$ Centre for Information Technology, Xi'an Technological University, China \\ ${ }^{2}$ School of Computer Science and Engineering, Xi'an Technological University, China
}

\author{
Article history \\ Received: 03-08-2020 \\ Revised: 07-09-2020 \\ Accepted: 09-09-2020 \\ Corresponding Author: \\ Baolong Liu \\ School of Computer Science \\ and Engineering, Xi'an \\ Technological University, \\ China \\ Email: b.liu@xatu.edu.cn
}

\begin{abstract}
Line laser rotation scanning can obtain accurate full contour information of the object with non-destructive testing. The key point in this process is the calibration rotation center. The calibration of the rotation center is mainly obtained through the characteristics of circles. Existing calibration schemes lead to a holed or over-gathered result. In order to solve this problem, this paper proposes a rotation hybrid calibration method to calibrate the rotation center. First, the data used to fit the center of the circle is screened by the symmetrical method to meet the requirements and then the points that meet the requirements are simulated by the least square method circle center. The experimental results show that the proposed method has a higher stability and accuracy compared to existing calibration methods and the calibration accuracy can reach $0.029 \mathrm{~mm}$.
\end{abstract}

Keywords: Non-Destructive Testing, Line Laser, Rotation Center, 3D Reconstruction

\section{Introduction}

Line laser is a non-contact measurement in threedimensional measurement with non-destructive testing and which has advantages such as high accuracy, fast speed, no damage, easy to control and automatic measurement (Kim et al., 2020). Line laser 3D measurement technology has broader prospects in reverse engineering, visualization, computer design, virtual reality, cultural relics protection, oral scanning and other fields. A laser transmitter, a rotating platform, two cameras and a microprocessor can be used to build a measurement system with high measurement accuracy with a low cost. According to different mechanical displacement platforms, there are two types of mechanical scanning: Translation platforms and rotary platforms. The contour information of the measured object can be obtained by rotating the platform. In online laser rotation scanning, the object rotates around a center point, which is the center of rotation. The calibration of the center of rotation is the main factor which restricts the accuracy of 3D measurement (Feng et al., 2020).

The rotation center was introduced in 3D reconstruction for obtaining the contour information of an object. Rotary scanning introduced circles into computer vision systems (Xia et al., 2009; Kinch et al., 2015), making the calibration of the center of rotation (center of the circle) a focus. If the result of fitting the center of the circle is inaccurate, the established contour model of the object will appear hollow or over-gathering. To solve this problem, Ying and Zha (2007) Constructed geometric constraints through the symmetry of a circle and proposed a contract center circle center. Liu and Su (2008) combined camera calibration and extraction of center-center projection points and repeated iterative calculations to improve the precise positioning of projection points. The disadvantage of this method leads to the large amount of calculations (Heikkila, 2000). Jiang and Quan (2005) chose a pair of concentric circles, starting from any point within the circle, gradually iteratively converging to fit the center of the circle, reducing this error. Chen and Zhao (2015) used three circles on different lines to locate the center of the circle. According to the relationship between the projection points of adjacent centers, the connection line between the centers was determined. The position of the center of the circle is the intersection of the connection lines 
(Zhenzhong and Guangjun, 2009; Pei-Cheng et al., 2007). This method is not applicable to some selfcalibration methods where the calibration block is a circle. Ziteng et al. (2015) proposed a method for locating the exact position of the projection point of the circle center from a circle in the imaging plane independently based on geometric constraints and invariant relationships in the projection transformation. Jiang et al. (2017) added the idea of random sampling consistency to the least squares method when fitting the rotation center and eliminated discrete points and edge points with large errors to ensure the accuracy of the center of the circle.

In order to improve the accuracy of rotation center calibration, this proposes a rotation hybrid calibration method to avoid excessive clustering problem in existing scheme. First, the data used to fit the center of the circle is screened for the points that meet the requirements through the symmetric method and then the points that meet the requirements are fitted to the center of the circle by the least square method. The calibration accuracy can reach $0.029 \mathrm{~mm}$, which higher than symmetric method, least squares method and light plane equation usually deployed.

In section 2, a brief description of measurement system is given. In section 3 , the calibration methods for the system are discussed in detail. Point cloud processing is described in section 4 . We make our conclusion in section 5 .

\section{Design and Analysis of Rotary Measurement System}

This article uses line laser dual triangulation to design and implement dental 3D reconstruction equipment, which is mainly used for 3D contour measurement of isolated teeth. It belongs to the traditional mechanical scanning structure and has the following characteristics: First, the device has a rotating platform, which can be completed by rotating scanning. Three-dimensional measurement of tooth models with complex morphology prevents the top of the measured object from being too large, blocking the bottom information and the rotating platform is placed at an angle; Secondly, the teeth are irradiated with line structured light. The polarization direction of line lasers is generally fixed, which allows the camera to capture line lasers containing dental information. Finally, the system uses double triangulation to Collecting data from different angles allows the point cloud data obtained from the left and right cameras to compensate each other, effectively eliminating blind spots and ensuring the integrity of the tooth model.

The hardware platform of this system is shown in Fig. 1. The main components include two industrial cameras, a line laser transmitter, a stepper motor, anectronically controlled rotating platform, a stage and a peripheral bracket.

The realization process of this article contains placing the teeth on the stage of the rotating platform, making the line structured light shine on a single tooth and controlling the rotating platform to rotate clockwise by the motor and the rotation angle is 2 degrees when collecting tooth data. The left and right cameras collect the reflected laser with tooth information and rotate to complete the scanning of the entire tooth. The left and right cameras collect 180 pictures each. The collected data is imported into a computer and the three-dimensional tooth contour is completed through image filtering, threshold segmentation, stripe center extraction, camera calibration, rotation platform calibration, data de-tilt and rotation stitching, point cloud reduction and point cloud stitching.

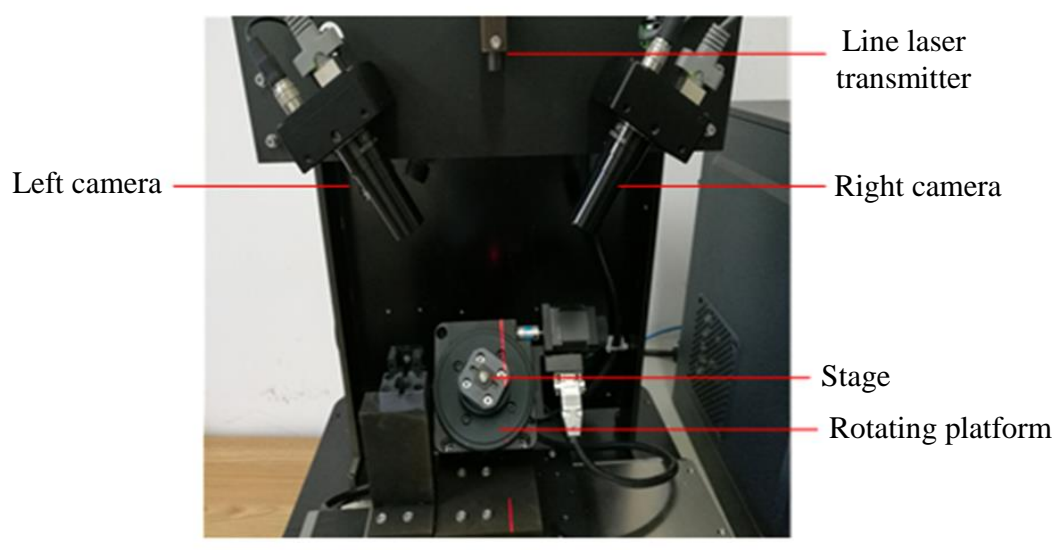

Fig. 1: Hardware platform diagram of the rotation system 


\section{Rotary Platform Calibration}

\section{Calibration of Rotation Center}

Generally speaking, the center of rotation is that in a plane, a figure rotates a certain angle around a point to obtain the change compared to another figure, where the point is the center of rotation (Qi, 2007).

During the rotation scanning, the measured object starts from a certain angle and starts to rotate $360^{\circ} \mathrm{C}$ around the rotation center. The rotation center of the rotation platform is unknown. In order to obtain the three-dimensional outline of the rotating object, the rotation center of the platform needs to be obtained by means of a calibration block or a sticking mark. After the rotation center is obtained, the subsequent de-tilt and rotation stitching can be performed. The fitted circle center is mainly divided into three cases: The actual circle center, greater than the actual circle center and smaller than the actual circle center. Greater than or less than the actual center of the circle will affect the accuracy of the final model. Therefore, the extraction of the rotation center is very critical.

The calibration block used for fitting the center of rotation in this study is shown in Fig. 2. In the middle is a white circular area.

\section{Fitting the Center of a Circle using the Symmetric Method}

The principle of fitting the circle center by the symmetric method is shown in Fig. 3, where $\theta$ represents the tilt angle of the rotating platform. On the premise that the rotation angle of the rotating platform is known, the equations of two straight lines $S_{1} S_{1}^{\prime}$, straight line $S_{2} S_{2}^{\prime}$ are fitted. The point $O$ is the center of rotation of the rotating platform and is also the intersection of two straight lines. Point $S_{1}$ is located at point $S_{1}^{\prime}$ after being rotated $180^{\circ} \mathrm{C}$ and point $S_{2}$ is located at point $S_{2}^{\prime}$ after being rotated $180^{\circ} \mathrm{C}$. The three points $S_{1}, O$ and $S_{1}^{\prime}$ are collinear, that is, symmetrical about the rotation center $O$ after the rotation and before the rotation. In actual data, due to the existence of the error, the intersection point of the two straight lines may be on the extension line of the two straight lines, or the two straight lines are parallel and there is no intersection point. Therefore, not all 10 sets of data collected can be used to fit the center of the circle. Therefore, according to this characteristic of the circle, the points that do not meet the requirements in the 10 sets of data can be deleted to ensure the accuracy of fitting the center of the circle.

\section{Least Squares Fitting Center}

The basic principle of the least square method find the best function match of a set of data and solve it by minimizing the sum of squared errors (Minglang and
Shunyi, 2013). The least square method is the simplest method to solve some absolutely unknown truth values. Using the least squares method to fit the center of the circle can reduce the error of fitting the center of the circle and ensure the accuracy of the center of the circle. The least square method is one of the mathematical optimization methods, which can minimize the sum of the squares of the circle center errors. The principle of least square fitting is shown in Fig. 4.

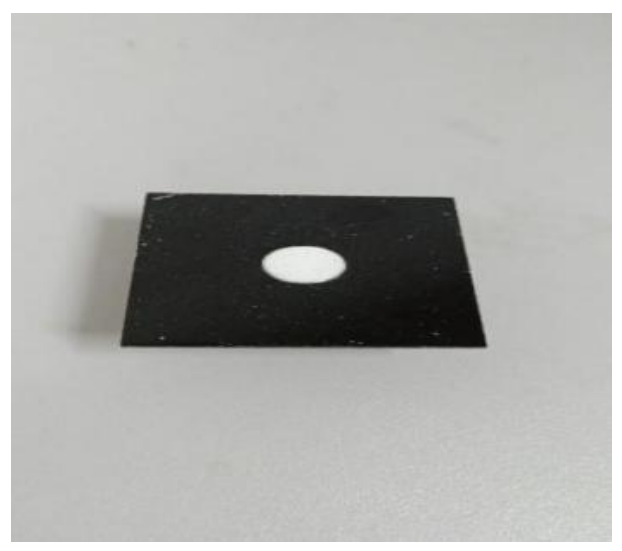

Fig. 2: Rotate the calibration block

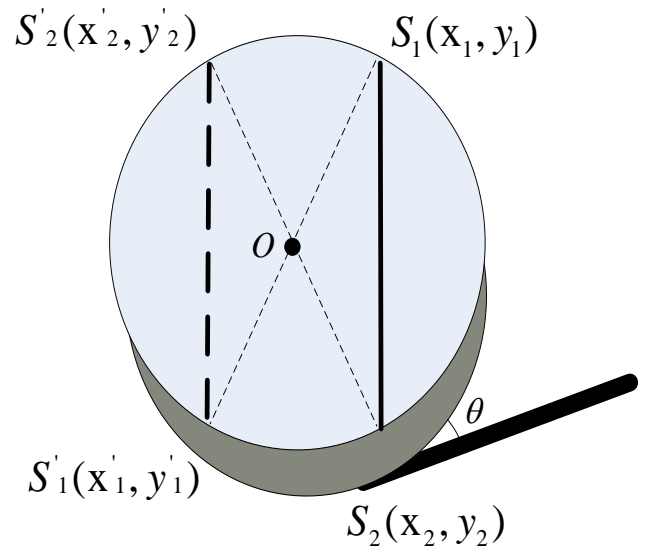

Fig. 3: Symmetric circle center fitting

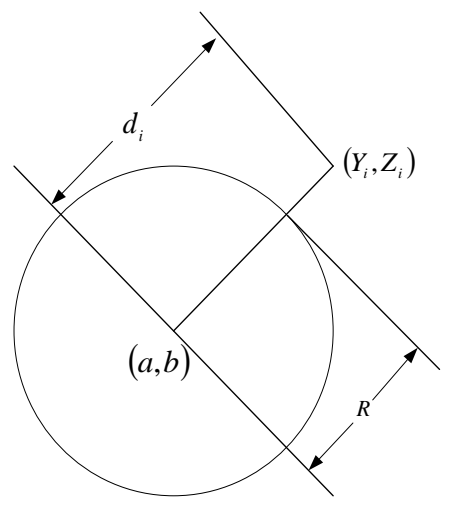

Fig. 4: Fitting the circle center by the least square method 
The specific process of using the least square method to fit the circle center is as follows

The standard equation for a known circle is shown in formula (1):

$$
R^{2}=(Y-a)^{2}+(Z-b)^{2}
$$

The general equation of a circle is shown in formula (2) and the general equation of a circle is transformed into a matrix form as shown in formula (3):

$Y^{2}+Z^{2}+D Y+E Z+F=0$

$\left[\begin{array}{lll}Y & Z & 1\end{array}\right] *\left[\begin{array}{l}D \\ E \\ F\end{array}\right]=-\left(Y^{2}+Z^{2}\right)$

The points that meet the requirements are selected by the symmetric method and the coordinates of the $Y$ and $Z$ axes of the points are known and the values of $D, E$ and $F$ are fitted by the least square method.

If $D^{2}+E^{2}-4 F^{2}>0$, then the center coordinates of the fit are $(-D / 2,-E / 2)$.

The radius of the circle is shown in formula (4):

$\frac{\sqrt{D^{2}+E^{2}-4 F}}{2}$

If $D^{2}+E^{2}-4 F^{2}=0$, it represents a point, the coordinates of the point are $(-D / 2,-E / 2)$.

If $D^{2}+E^{2}-4 F^{2}<0$, it means that the fitted figure is not a circle.

\section{Light Plane Equation Fitting Circle Center}

In the process of $3 \mathrm{D}$ reconstruction by rotating scanning, the light plane fitting is performed on the point cloud set of calibration blocks obtained by the camera and the rotation center of the rotating platform can be fitted (Wang and Ding, 2012; Chen et al., 2013). The line laser transmitter emits a line of laser light. The line laser will intersect the surface of the calibration block to form a plane. This plane is called the light plane. As shown in Figure 5, point $A$ in the figure is the line laser emitter and the straight line is the intersection line formed by the line laser and the surface of the calibration block. The plane formed by the three points $A, B$ and $C$ is the light plane. The points in the light plane $(\triangle A B C)$ all conform to the light plane equation.

During the online structured light rotation scan, the point clouds obtained by the camera are in the light plane, so all point clouds conform to the light plane Equation (5):

$$
a x+b y+c z+d=0
$$

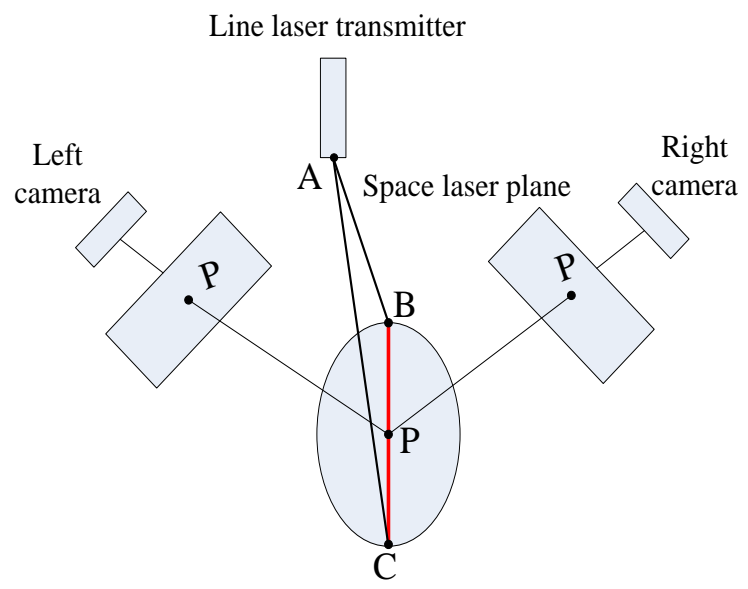

Fig. 5: Light plane equation

The point cloud data obtained by scanning the rotating platform are sequentially brought into the light plane equation of the above formula to obtain formula (6). The values of the four unknown $a, b, c$ and $d$ can be solved by least squares fitting:

$\left\{\begin{array}{c}a x_{1}+b y_{1}+c z_{1}+d=0 \\ a x_{2}+b y_{2}+c z_{2}+d=0 \\ \vdots \\ a x_{n}+b y_{n}+c z_{n}+d=0\end{array}\right.$

The rotation center of the rotating platform can be expressed as formula (7):

$O=\left(\frac{1}{n} \sum_{i=1}^{n} x_{i}, \frac{1}{n} \sum_{i=1}^{n} y_{i}, \frac{1}{n} \sum_{i=1}^{n} z_{i}\right)$

\section{Rotary Hybrid Calibration}

In this study, a rotation hybrid calibration method is proposed to fit the circle center as the rotation center of the rotation platform. The method first uses 10 groups of data to select the data that meets the circle by using the symmetry method and then uses the least squares method to fit the data that meets the requirements for circle center fitting. If the data used to fit the center of the circle does not satisfy the symmetry method, which means that this point is not a point on the circle. If it is directly used to fit the center of the circle, the accuracy of the tooth model will be reduced. The tooth model appears cavitation or excessive coincidence, so the center of the circle cannot be fitted directly by the symmetrical method or the least square method.

The fitting process of the rotation center is as follows. The world coordinates of 10 sets of rotation calibration blocks are selected by the method of symmetry to meet the characteristics of the circle. The least square method is deployed to fit the center of the world coordinates to 
meet the requirements. The fitting result of the symmetric method is shown in Fig. 6a. Connecting the four points separately, there is an intersection between the two lines and the intersection point is not on the extension of the line. User can use this set of data to fit the center of the circle. From Fig. $6 \mathrm{~b}$, it can be seen that there is no intersection point between the two straight lines. The intersection point may be on the extension of the two straight lines or the two straight lines are parallel. This set of data does not meet the requirements and cannot be used to fit the center of the circle. Through this feature of the circle, the points do not meet the requirements in the data eliminated, thereby ensuring the accuracy of the center of the circle.

In Fig. 6a, the point $" * "$ is the starting point and the points connected by the scattered dotted lines are compared. The point " $+"$ is a symmetrical point after being rotated 180 degrees and a point connected by a tighter dotted line, unit:mm. Figure $6 \mathrm{~b}$ indicates data that does not meet the requirements. There is no intersection between the two straight lines or the intersection is on the extension line, unit:mm.

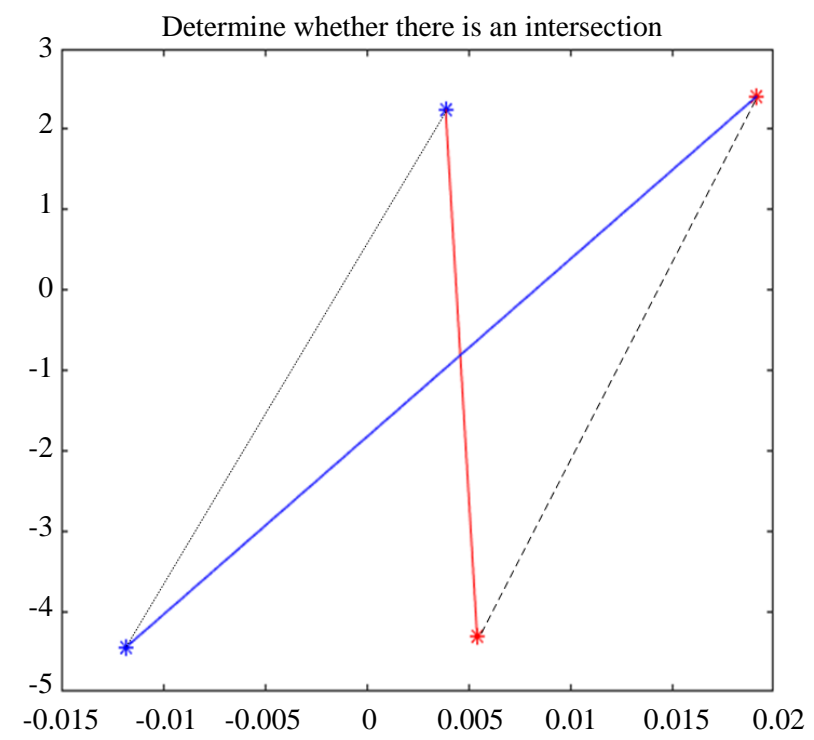

(a)

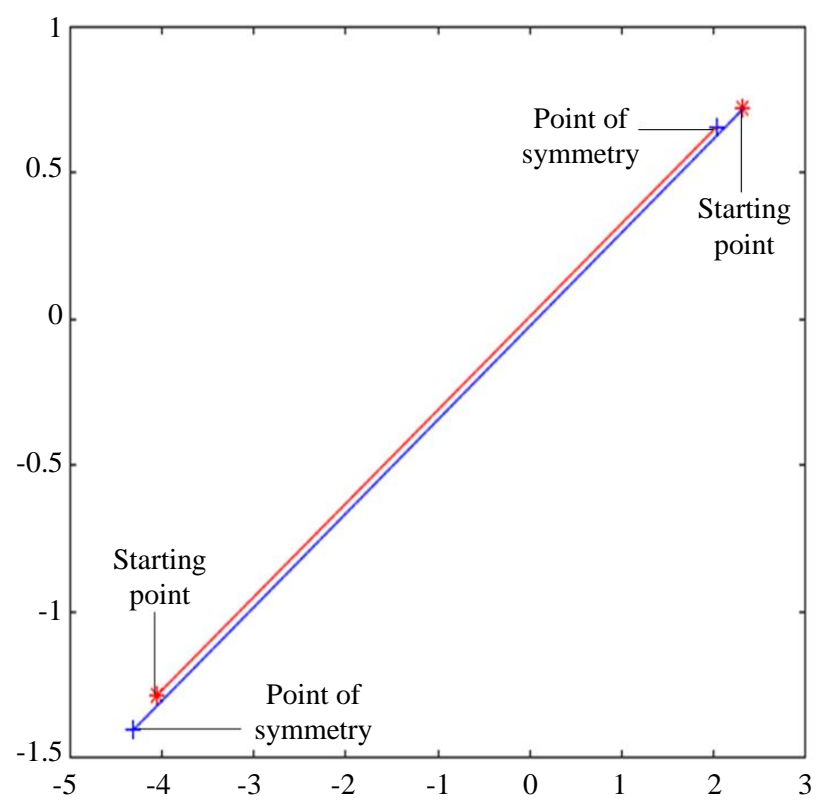

(b)

Fig. 6: The result of fitting the center of the circle by the symmetric method 
After removing the error points, the remaining points are fitted to the center of the circle by the method of least squares. The fit result of the center of the circle is shown in Fig. 7.

In Fig. 7, where the "o" point is the starting point, that is, the point connected by the solid line. The "*" point is a symmetrical point after being rotated $180^{\circ} \mathrm{C}$, that is, a point connected by a dotted line. The "+" indicates the center of the fit. The green circle represents the circle drawn by the fitted center and radius. The circle in the figure is because the 10 groups used to calculate the center of rotation are all displayed. unit:mm.

\section{Analysis of Calibration Results of Rotation Center}

The results of the fitting center methods above are shown in Table 1 and the comparison results are shown in Fig. 8. Compared to the symmetrical method of fitting the center of the circle, the rotating hybrid calibration method improves the fitting accuracy by $1.2 \%$. Compared to the least square method, the fitting accuracy improves by $0.98 \%$. Compared to the light plane equation, the fitting accuracy improves by $0.38 \%$. The figure shows the comparison results of the four fitting methods 10 times. It can be seen from the figure that the center of the circle fitted by the symmetric method and the least square method is slightly larger than the rotation mixed calibration method and the center of the light plane equation fitting is unstable. In general, the circle center of the hybrid calibration fit is more stable and closer to the actual rotation center.
In order to evaluate the method proposed, a comparison is made with four different method including symmetric method, least squares method, light plane equation and hybrid calibration method presented in this study as shown in Fig. 9. Figure 9a shows the top view of a tooth model created by fitting the center of the circle using a symmetric method and Fig. $9 \mathrm{~b}$ shows the top view of a tooth model created by fitting the center of the circle by the least square method. It can be seen that the tooth model established by these two methods has holes as marked in Fig. 9a and $9 \mathrm{~b}$. The holes will lead to a failed 3D model establishment. In fact, the larger the gap between the fitted center and the actual center is, the larger the hole in the middle of established model (Wu et al., 2020; Lu et al., 2020). In order to observe the clustering phenomenon, Figure 9c shows the side view of tooth model established by fitting the center of the light plane equation to the center of the circle. Since the center of the fitted circle is sometimes slightly smaller than the actual center of the circle, which usually leads to a excessive convergence ( $\mathrm{Lu}$ et al., 2020). Figure 9d shows the tooth model created by fitting the center of the circle with the rotation hybrid calibration method. It can be seen that the center of the tooth is the closest to the actual center. The tooth model created has no holes and there is no excessive convergence. This can prove the correctness of this method and the accuracy of fitting the center of the circle.

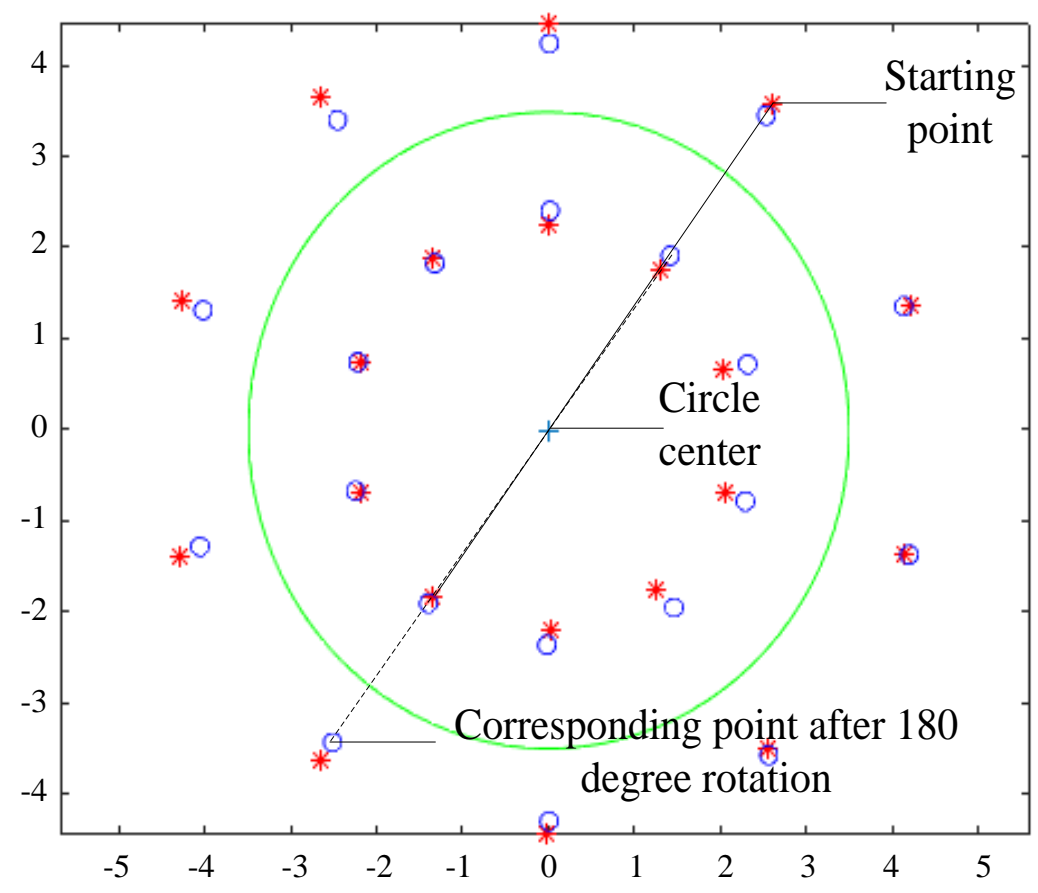

Fig. 7: Center fit of the least square method 


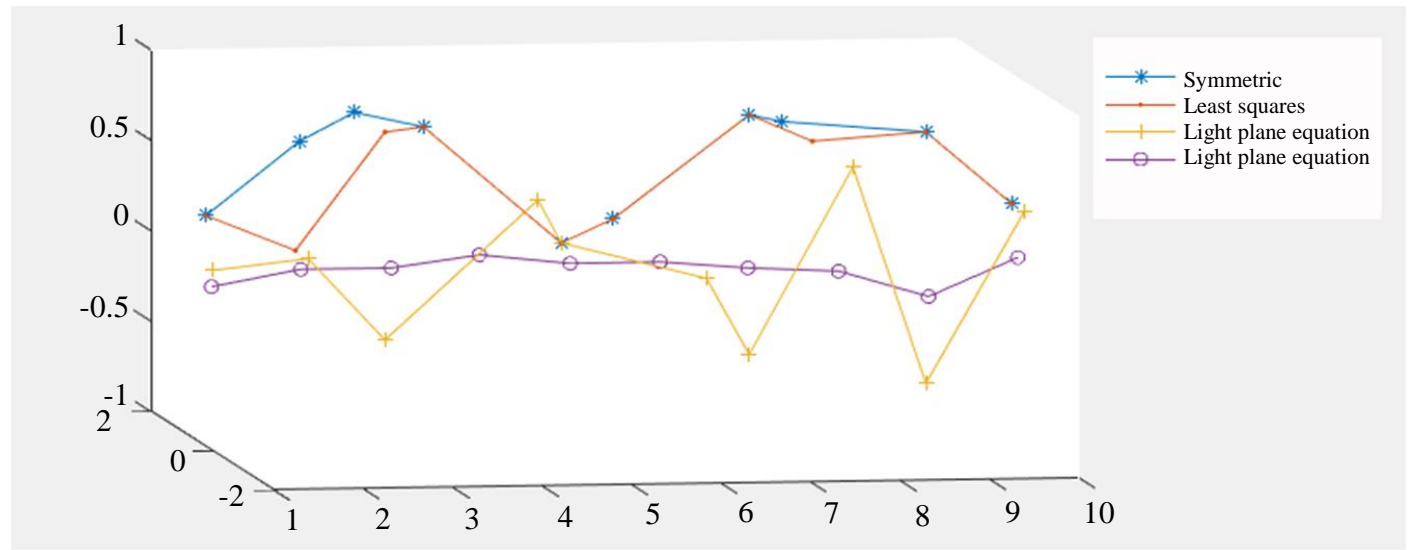

Fig. 8: Comparison of circle center fitting

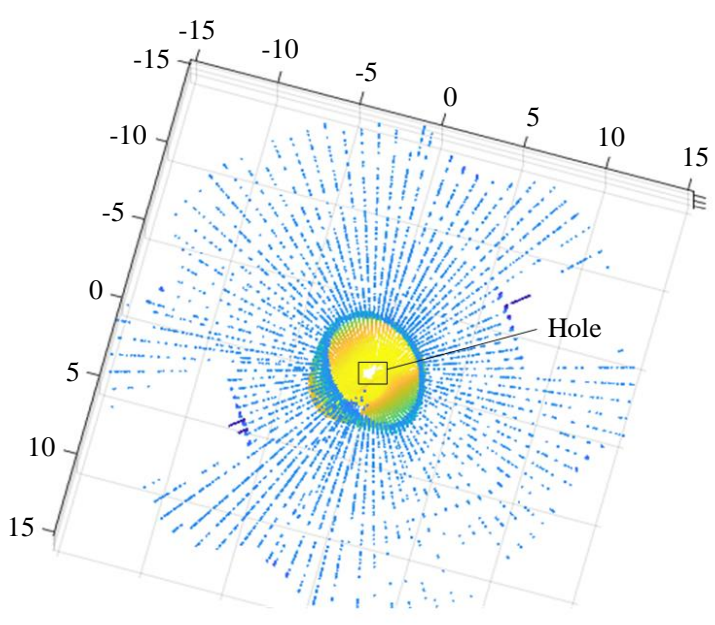

(a)

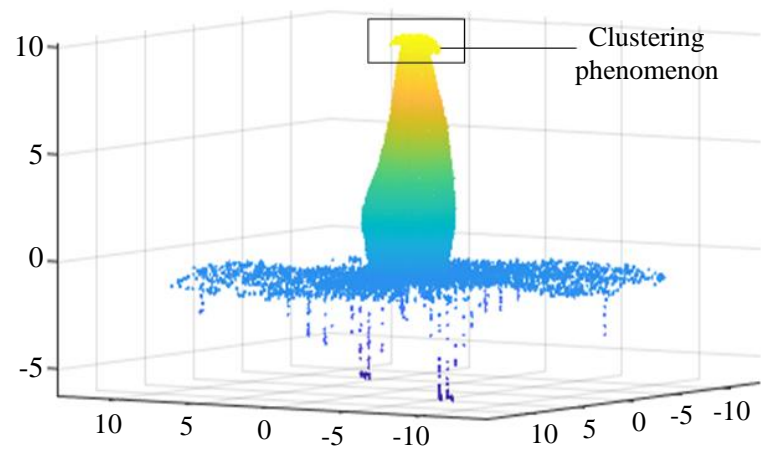

(c)

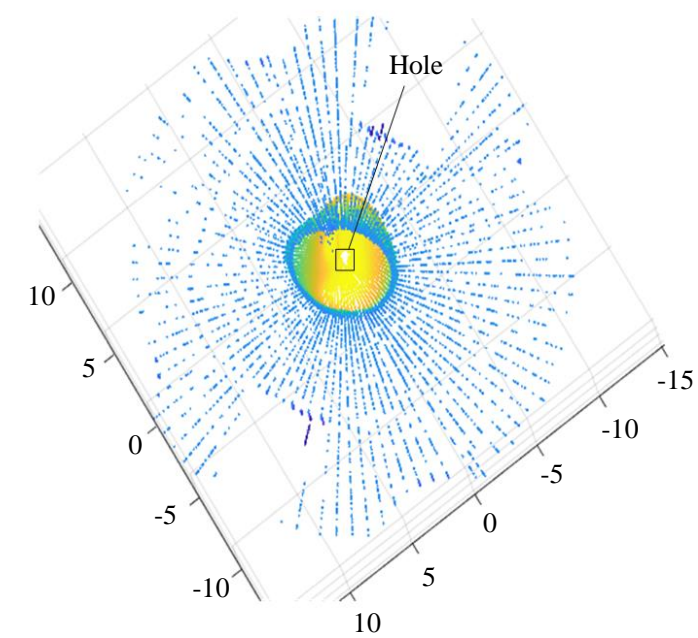

(b)

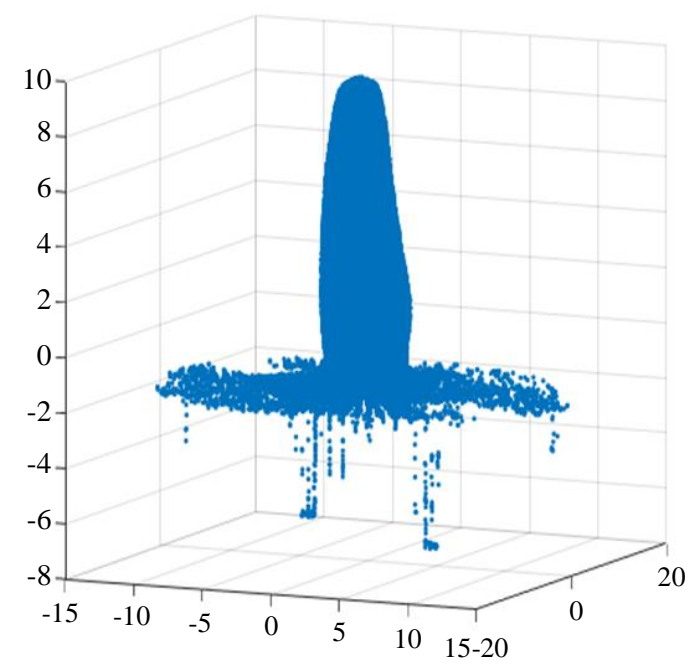

(d)

Fig. 9: Comparison model of fitting circle center (unit:mm) (a) Top view of a symmetric tooth model (b) Top view of a least squares tooth model (c) Side view of tooth model with light plane equation (d) Side view of the model with hybrid calibration method 
Table 1: Fitting the center of the circle: (unit:mm)

\begin{tabular}{lllll}
\hline Fitting times & Symmetric & Least squares & Light plane equation & Rotary hybrid calibration \\
\hline 1 & $(0.2078,0.2777)$ & $(0.2078,0.2776)$ & $(-0.0001,-0.0029)$ & $(0.0280,-0.0960)$ \\
2 & $(0.0799,0.6951)$ & $(0.2063,0.0772)$ & $(-0.2063,0.0772)$ & $(0.0323,-0.0072)$ \\
3 & $(1.2071,0.7248)$ & $(0.2069,0.7250)$ & $(0.2071,-0.4248)$ & $(0.0071,-0.0048)$ \\
4 & $(1.8568,0.5666)$ & $(1.8566,0.5665)$ & $(-1.8568,0.5666)$ & $(0.0456,0.0561)$ \\
5 & $(0.3009,0.0872)$ & $(0.3010,0.0871)$ & $(0.3008,0.0872)$ & $(0.0036,0.0057)$ \\
6 & $(1.5140,0.0806)$ & $(1.5139,0.0805)$ & $(-1.5139,0.0849)$ & $(-0.0051,0.0080)$ \\
7 & $(0.0046,0.8125)$ & $(0.0045,0.8124)$ & $(0.0046,-0.5125)$ & $(0.0460,-0.0381)$ \\
8 & $(1.8568,0.5665)$ & $(0.8568,0.5665)$ & $(-0.4568,0.5664)$ & $(0.0058,-0.0591)$ \\
9 & $(0.0799,0.6951)$ & $(0.0798,0.6949)$ & $(0.0799,-0.6947)$ & $(-0.0074,-0.2051)$ \\
10 & $(0.2088,0.2776)$ & $(0.2078,0.2777)$ & $(-0.2080,0.2780)$ & $(-0.0020,0.0027)$ \\
\hline
\end{tabular}

\section{Calibration of Tilt Angle}

In order to achieve a full scan of the measured object, the rotary platform is often designed to maintain a certain tilt angle with the plane formed between the world coordinate $X$ axis and $Y$ axis. If the tilting angle of the rotating object platform is not designed, which indicates that the line laser directly irradiates the measured object directly and it will have a certain degree of influence on the height of the tooth model. Because the teeth have a certain arc, as shown in Fig. 10, if the measured teeth are scanned vertically, the tooth width of area $A$ is larger than that of area B and area A will block the area B, which will cause the area B of the tooth model. Some data are missed. Therefore, a certain degree of tilt angle is added to the rotating platform when the scanning device is designed. In order to obtain a three-dimensional model of the scanned object, it is also necessary to calibrate the tilt angle of the rotating platform and restore the scan data to a state without a tilt angle.

The calibration of the tilt angle of the rotating platform is mainly realized by rotating the data of the calibration block. The data is used to fit the center of the circle select the two endpoints of the calibration block data and the data to fit the tilt angle is implemented using the entire laser fringe data. Since the data of the $Y$ axis and the $\mathrm{Z}$ axis are known, the data is first fitted to a straight line, the slope of the straight line is solved and then the slope of the straight line is converted into the tilt angle of the rotating platform.

The specific steps to calculate the tilt angle of the rotating platform are as follows:

Step1: Fit the space points of the rotation calibration block on the rotation platform to a straight line;

Step2: Find the slope of the fitted straight line. The matrix form of the fitted straight line is shown in the following formula (8), where $k$ is the slope of the straight line and $b$ is the translation distance:

$\left[\begin{array}{ll}y_{i} & 1\end{array}\right] *\left[\begin{array}{l}k \\ b\end{array}\right]=z_{i}$

Step3: The slope of the fitted straight line is the tangent of the tilt angle of the rotating platform

Step4: Turn the tangent value into radian

Step5: Tilt angle $\beta=$ radian*180/3.1415926.

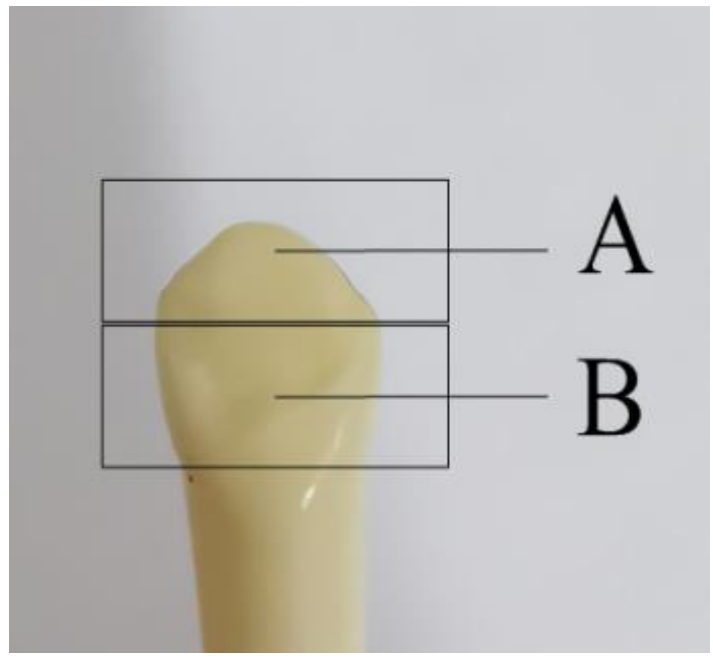

Fig. 10: Tooth occlusion

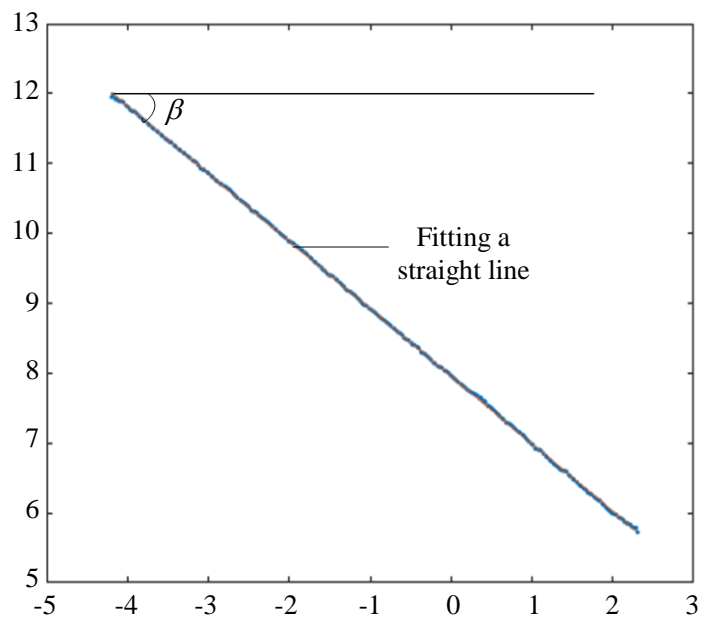

Fig. 11: Fitting graph of tilt angle of calibration block data: Where $\beta$ represents the tilt angle of the rotating platform

Through method above, the tilt angle of the rotating platform can be fitted. The result of fitting a straight line in the space of the rotated calibration block is shown in Fig. 11. Finally, the tilt angle of the rotating platform was fitted: $-44.1205^{\circ} \mathrm{C}$. 


\section{Point Cloud Processing}

\section{Point Cloud Acquisition}

The resolution of the camera used for data acquisition in this study is $1280 * 1024 \mathrm{dpi}$ and the effective field of view is $20 * 18 \mathrm{~mm}$.

The data collection process is as follows:

Step1: Adjusting the rotating platform to the working state, placing a rotating calibration block or tooth model on the rotating platform carrier and adjusting the position of the rotating platform by left and right translation so that the line structure light passes through the center of the rotating platform.

Step2: Rotating the rotating platform clockwise and the camera starts to collect data. The camera takes a picture every time the rotating platform rotates $36^{\circ} \mathrm{C}$. The camera takes a picture every time the rotating platform rotates $2^{\circ} \mathrm{C}$ to obtain dental data collection.

Step3: The system performs filtering, threshold segmentation and other processing on the captured pictures and then extract the center coordinates of the laser stripes in the pictures taken by the left and right cameras as pixel coordinates.

Step4: Calculate the world coordinates corresponding to the pixel coordinate system and store them in the sample set.

Step5: After rotating $360^{\circ} \mathrm{C}$, export the sample set to calculate the parameters of the rotating platform and establish the tooth model.

The point cloud data collected by the camera is shown in Fig. 12.

\section{Point Cloud Declination}

After calibrated the tilt angle and the rotation center of the rotation platform, it is necessary to perform de-tilt angle for the tooth data converted to world coordinates. The point cloud declination is simply the data obtained by rotating the point cloud data around the rotation center by an inclination angle. Generally speaking, de-tilt is to change the tilt angle of the rotating platform to 0 degrees, which uses the rotation center as the coordinate origin and restore the collected point cloud data to a state without a tilt angle.

The point cloud data is de-tilted to arrange it into a matrix form as shown in formula (9), where $\beta$ represents the tilt angle of the rotating platform and $(-y r,-z r)$ represents the center of rotation of the rotating platform:

$$
\left[\begin{array}{l}
y_{q} \\
z_{q}
\end{array}\right]=\left[\begin{array}{ccc}
\cos \beta & -\sin \beta & -y r \\
\sin \beta & \cos \beta & -z r
\end{array}\right] *\left[\begin{array}{c}
Y_{w} \\
Z_{w} \\
1
\end{array}\right]
$$

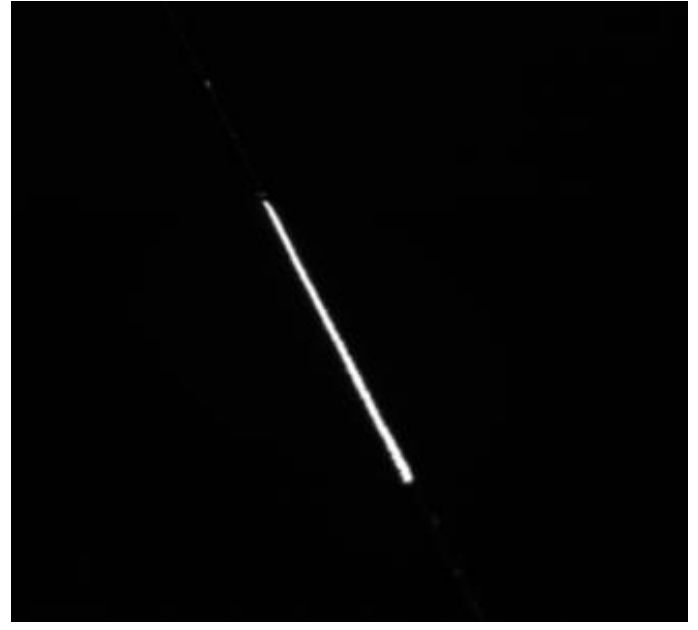

Fig. 12: Raw data collected

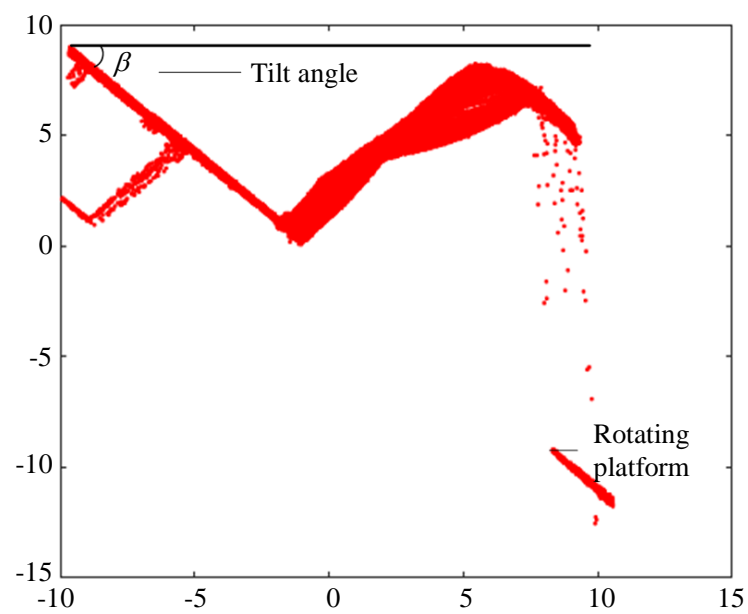

Fig. 13: Before tooth data de-tilt: where $\beta$ represents the tilt angle of the rotating platform, unit:mm

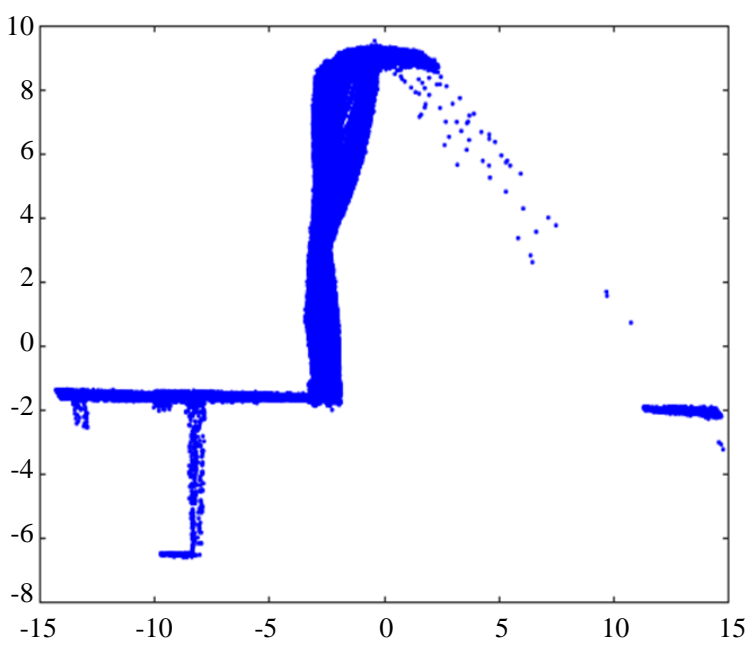

Fig. 14: After tooth data de-tilt 
The tooth point cloud data before de-tilting is shown in Fig. 13. The tooth point cloud data is de-tilted shown in Fig. 14. It can be seen that the tooth data has been restored to the state without a tilt angle, unit:mm.

\section{Point Cloud Rotation Stitching}

Rotating stitching is to de-tilt the tooth cloud data collected each time and then it combines the angles of each rotation of the rotating platform to obtain the final point cloud data. When collected dental data, the rotating platform rotates $2^{\circ} \mathrm{C}$ and the camera collects the data once. Because the data in the pictures collected by the camera are in their respective local coordinates, the data in these pictures need to be unified into the same coordinate system by rotation and stitching. Before the process is completed, tooth data has been converted from pixel coordinates to world coordinates and after all data is unified to the same coordinate, a complete tooth model can be completed. This process is called rotating stitching.

The matrix form of point cloud rotation stitching is shown in formula (10), where $\theta$ represents the rotation angle when the rotation platform collects data. $y_{q}$ and $z_{q}$ represent the point cloud data after de-tilt angle. $x_{w r}, y_{w r}$ and $z_{w r}$ represent the actual point cloud data after rotation and stitching:

$$
\left[\begin{array}{l}
x_{w r} \\
y_{w r} \\
z_{w r}
\end{array}\right]^{T}=\left[\begin{array}{ll}
y_{q} & z_{q}
\end{array}\right] *\left[\begin{array}{ccc}
-\sin \theta & \cos \theta & 0 \\
0 & 0 & 1
\end{array}\right]
$$

The rotation stitching results of the tooth data are shown in Fig. 15. Compared to Fig. 14 after de-tilt angle, the data coordinates in 180 pictures are unified and a single tooth model is obtained, unit:mm.

\section{Point Cloud Streamlining}

Point cloud reduction is to reduce the tooth model after rotation and splicing to a certain extent and improves the speed of subsequent point cloud processing. The further development of laser scanning technology resulted in more and more high-precision point clouds acquired on the surface of objects, which intensifies the complexity of point cloud data processing. If the point cloud data is not simplified, it will consume a lot of computational resources during the $3 \mathrm{D}$ reconstruction process, increase the processing time of the point cloud data and reduce the efficiency of establishing the 3D model of the measured object. Therefore, streamlining point cloud data is necessary to $3 \mathrm{D}$ modeling.

The point cloud reduction results are shown in Table 2 and Fig. 16. Table 2 can directly observe the reduction in the amount of point cloud data. Figure 16a shows the original point cloud, that is, the state of the point cloud is not simplified. Figure 16b shows the initial streamlining of the point cloud, deleting insignificant data such as the scanned rotating platform, leaving only the tooth data part. Figure 16c shows that on the basis of Fig. 16b, the point cloud is simplified and the characteristic part of the point cloud is retained. Triangulate to speed up streamlining increases the processing speed of subsequent point clouds.

\section{Measurement and Evaluation of Tooth Model}

In order to make the established tooth model more in line with the 3D scene and clearly displaying the detailed information such as tooth structure, the stitched point cloud data also needs to be rendered. After rendered the tooth model, the laser point cloud is clearly visible and the texture details are interleaved brightly and darkly, which highlights the three-dimensional structure of the tooth model. This article uses Open GL to render the tooth model. The correctness of the calibration results can be visually verified through the rendering results, so that the scene information is effectively enhanced. The tooth model rendered by OpenGL is shown in Fig. 17 and the original tooth image is shown in Fig. 17c. After rendered, the outline information and details of the teeth can be observed clearly and the depth information of the tooth model is obtained. The depth of the tooth in Fig. 17 is $13.79 \mathrm{~mm}$.

Table 2: Streamline of point cloud: (unit: pcs)

\begin{tabular}{lll}
\hline & Left camera & Right camera \\
\hline Raw data & 53086 & 58151 \\
Initial streamlining & 38360 & 39415 \\
Delaunay Simplify triangulation & 12876 & 13158 \\
\hline
\end{tabular}

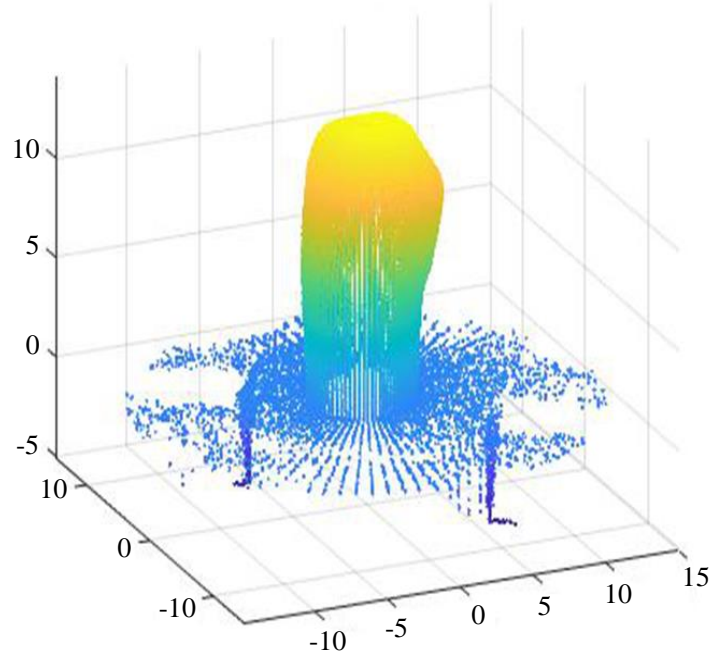

Fig. 15: Rotating and stitching results of dental data 


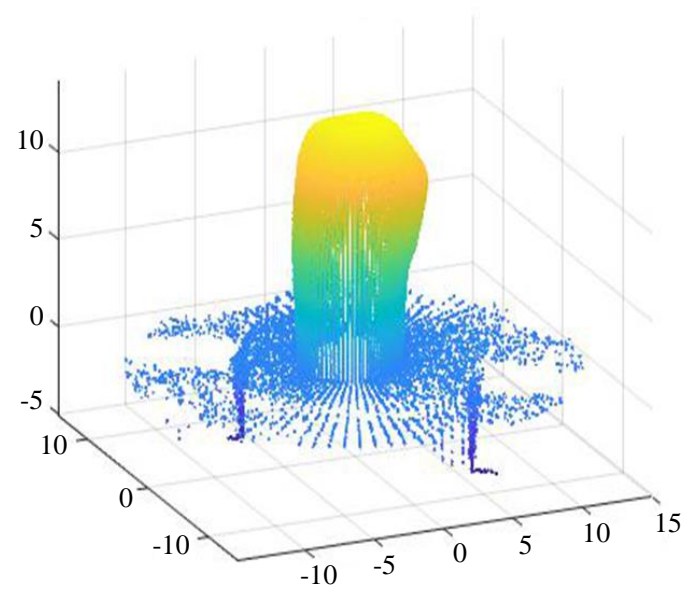

(a)

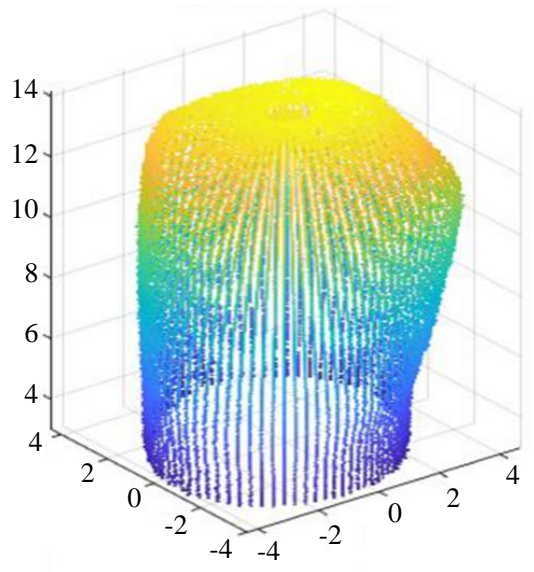

(b)

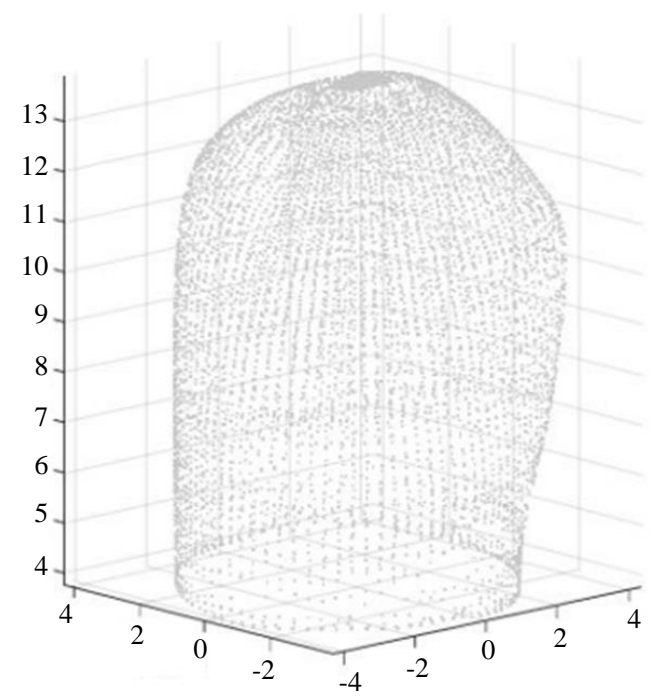

Fig. 16: Simplification of tooth point cloud data (unit:mm) (a) Before point cloud streamlining (b) Preliminary point cloud streamlining (c) After streamlining the point cloud

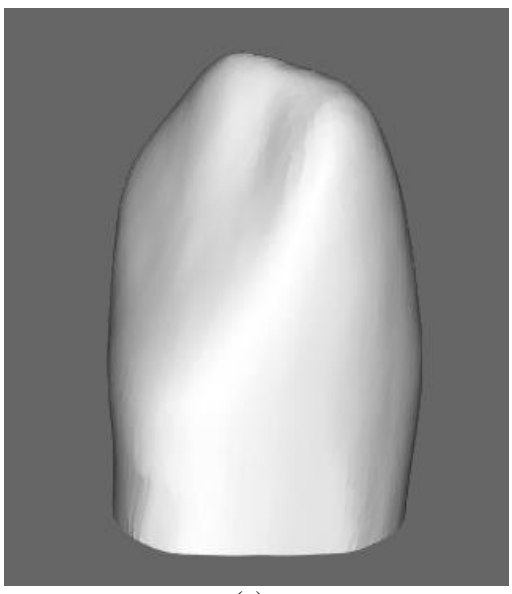

(a)

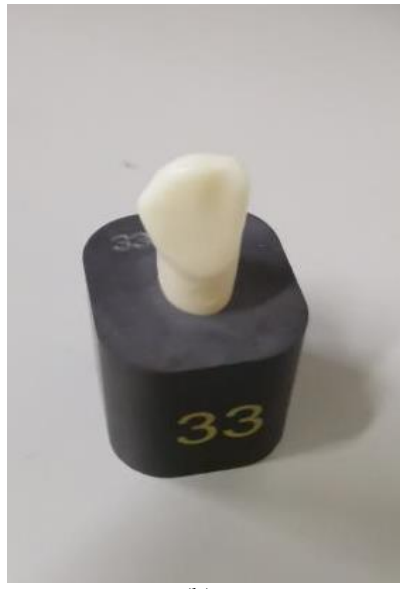

(b)

Fig. 17: The rendering result of the tooth model (a) Teeth rendering (b) Original tooth 


\section{Conclusion}

This paper mainly introduces the reconstruction process of denture rotation scanning. It mainly includes three parts: System design, calibration of rotating platform and point cloud processing. The rotation platform calibration is to calibrate the rotation center and tilt angle of the rotation platform. In this study, a rotation hybrid calibration method is proposed to calibrate the center of rotation. The points that meet the requirements are selected by the symmetrical method. It is used to fit the center of the circle by least squares. This method guarantees accurate circle center. The experimental results show that the calibration result of this method is stable and the calibration accuracy is satisfied. The calibration process of the tilt angle of the rotating platform is to fit the calibration block data as a straight line, find the slope of the straight line and obtain the tilt angle of the rotating platform by the slope. After calibrated the rotation center and tilt angle, it is necessary to perform de-tilt and rotation stitching on the collected point cloud data. De-tilt is to move the coordinate origin to the center of rotation and restore the point cloud to a state without tilt angle. Rotating stitching is mainly to unify the point clouds of each rotation angle into the same coordinate system and stitch the laser stripes from the world's three-dimensional coordinates into a tooth model. The tooth model finally established can intuitively prove the correctness and stability of the algorithm proposed in this study.

\section{Acknowledgement}

The present work is supported by the fund of Science and Technology Plan Project of Weiyang District, Xi'an (Project No: 201836).

\section{Author's Contributions}

Yi Guo: Has conceived and designed the experiments, data analysis, manuscript writing and publication.

Doudou Lei: Critical revision of the article.

Yu Liu: Has reviewed and revised the manuscript.

Baolong Liu: Final approval of article

\section{Ethics}

Authors should address any ethical issues that may arise after the publication of this manuscript.

\section{Reference}

Chen, X. Y., Ma, Z., Hu, Y., Chen, Y. Q., \& Bi, F. L. (2013). A new method for accurate location of concentric circles in visual measurement. J. Optoelectron. Laser, 24, 1524-1528.
Chen, X., \& Zhao, Y. (2015). A linear approach for determining camera intrinsic parameters using tangent circles. Multimedia Tools and Applications, 74(15), 5709-5723.

Feng, W., Su, Z., Han, Y., Liu, H., Yu, Q., Liu, S., \& Zhang, D. (2020). Inertial measurement unit aided extrinsic parameters calibration for stereo vision systems. Optics and Lasers in Engineering, 134, 106252 .

Heikkila, J. (2000). Geometric camera calibration using circular control points. IEEE Transactions on pattern analysis and machine intelligence, 22(10), 1066-1077.

Jiang, G., \& Quan, L. (2005, October). Detection of concentric circles for camera calibration. In Tenth IEEE International Conference on Computer Vision (ICCV'05) Volume 1 (Vol. 1, pp. 333-340). IEEE.

Jiang, Y., Liwei, T., \& Shijie, D. (2017). Automatic center extraction and sorting based on improved circular target. Computer Engineering and Applications, 53(19), 246-251.

Kim, J. A., Kim, J. W., Kang, C. S., Lee, J. Y., \& Jin, J. (2020). On-machine calibration of angular position and runout of a precision rotation stage using two absolute position sensors. Measurement, 153, 107399.

Kinch, K. M., Bell III, J. F., Goetz, W., Johnson, J. R., Joseph, J., Madsen, M. B., \& Sohl-Dickstein, J. (2015). Dust deposition on the decks of the Mars Exploration Rovers: 10 years of dust dynamics on the Panoramic Camera calibration targets. Earth and Space Science, 2(5), 144-172.

Liu, Q., \& Su, H. (2008, May). Correction of the asymmetrical circular projection in DLT camera calibration. In 2008 Congress on Image and Signal Processing (Vol. 2, pp. 344-348). IEEE.

Lu, J., Cai, Z., Yao, B., Cai, S., Ma, X., \& Liu, W. (2020). Error compensation and accuracy analysis of laser measurement system based on laser-beam calibration. Optik, 200, 163272.

Minglang, Z., \& Shunyi, Z. (2013). Registration method of point cloud data of rotating platform. Journal of Surveying and Mapping, 42(1), 73-79.

Pei-Cheng, H. U., Ning, L. I., \& Jian-jiang, Z. H. O. U. (2007). Improved camera self-calibration method based on circular points. Opto-Electronic Engineering, 34(12), 54-60.

Qi, W. (2017). 3D reconstruction technology based on structured light and rotating platform [D]. University of Electronic Science and Technology.

Wang, H. R., \& Ding, W. (2012). A Novel Camera Calibration Method Based on Circle Array Target. In Advanced Materials Research (Vol. 472, pp. 968-973). Trans Tech Publications Ltd. 
Wu, X., Tang, N., Liu, B., \& Long, Z. (2020). A novel high precise laser 3D profile scanning method with flexible calibration. Optics and Lasers in Engineering, 132, 105938.

Xia, R. B., Liu, W. J., Zhao, J. B., \& Xu, J. T. (2009). Fully automatic camera calibration method based on circular markers. Chinese Journal of Scientific Instrument, 30(2), 368-373.

Ying, X., \& Zha, H. (2007, September). An efficient method for the detection of projected concentric circles. In 2007 IEEE International Conference on Image Processing (Vol. 6, pp. VI-560). IEEE.
Zhenzhong, W., \& Guangjun, Z. (2009, November). Estimation of projected circle centers from array circles and its application in camera calibration. In 2009 Asia-Pacific Conference on Computational Intelligence and Industrial Applications (PACIIA) (Vol. 1, pp. 182-185). IEEE.

Ziteng, L., Ruiling, W., \& Qiuping, W. (2015). Accurate positioning of the center projection point in visual calibration. Progress in Laser and Optoelectronics, $52(9), 091001$. 